\title{
Mass of the Stabilized Radion in the Limit of Finite Quartic Coupling
}

\author{
Ali Tofighi' ${ }^{1}$ and Vahid Reza Shajiee ${ }^{2}$ \\ ${ }^{1}$ Department of Physics, Faculty of Basic Science, University of Mazandaran, P.O. Box 47416-95447, Babolsar, Iran \\ ${ }^{2}$ Young Researchers and Elite Club, Mashhad Branch, Islamic Azad University, Mashhad, Iran \\ Correspondence should be addressed to Vahid Reza Shajiee; v.shajiee@mshdiau.ac.ir
}

Received 28 September 2016; Revised 6 February 2017; Accepted 1 March 2017; Published 12 March 2017

Academic Editor: Edward Sarkisyan-Grinbaum

Copyright (c) 2017 Ali Tofighi and Vahid Reza Shajiee. This is an open access article distributed under the Creative Commons Attribution License, which permits unrestricted use, distribution, and reproduction in any medium, provided the original work is properly cited. The publication of this article was funded by SCOAP ${ }^{3}$.

We present an exact treatment of the modulus stabilization condition with the general boundary conditions of the bulk scalar field in the Randall-Sundrum model. We find analytical expressions for the value of the modulus and the mass of the radion.

\section{Introduction}

The hierarchy problem is one of the most attractive open problems in the modern physics. Roughly speaking, the problem is the large discrepancy between the weak and the Planck scale. The problem was addressed by several theories, like supersymmetry, and higher dimensional theories; however, it has remained unsolved in the literature. Through the efforts in this direction, an impressive work is Randall-Sundrum (RS) model [1] which introduces a small extra dimension. The model has two branes which are called "Planck" and "TeV" branes; also it is assumed that a slice of $\mathrm{AdS}_{5}$ spacetime exists between the branes. A five-dimensional solution to the Einstein field equations in RS model is given by

$$
d s^{2}=e^{-2 k r_{c}|\phi|} \eta_{\mu \nu} d x^{\mu} d x^{\nu}-r_{c}^{2} d \phi^{2},
$$

where $-\pi \leq \phi \leq \pi$ is the extra dimension coordinate, the coefficient $r_{c}$ is the compactification radius, and the parameter $k$ is related to the 5-D Planck mass, $M$.

It would be interesting to check out the stability of the extra dimension, $\phi$, in the RS model. Such an investigation was addressed by Goldberger and Wise (GW) [2]. The GW model contains a massive scalar field with usual kinetic term in the bulk and quartic interactions localized on the branes. The original work of [2] has several deficiencies, namely:

(1) The bulk mass term breaks the conformal invariance of the theory.
(2) In the limit of infinite quartic coupling considered in [2], it is not possible to unravel the complete structure of the critical points of the theory; hence they miss the source of instability as indicated by the existence of a closely spaced maximum.

(3) The boundary conditions of the model are comprised of a pair of coupled cubic algebraic equations. In [2] these boundary conditions are not solved; instead they merely choose a specific configuration.

(4) In addition they only consider the leading order term in their calculations. Hence their treatment of the subject matter is an approximate one.

Therefore, many studies were done on this subject [3-12]. In [3-5], the authors have considered the stabilization of the modulus containing a scalar field, which interacts with the spacetime curvature $R$, in the bulk. These theories have conformal invariance at certain value of the coupling constant of the curvature and the scalar field; hence they remedy objection 1 , raised above.

An exact analysis of the GW mechanism has been discussed in [6] and the objections 2-4 have been addressed. However their treatment of the stabilized modulus is a numerical one. They also do not address the issue of the mass of radion at finite values of the quartic coupling constant. The issue of Goldberger-Wise mechanism with the general boundary conditions also has been discussed in 
[13]. Recently, the issue of stability of the Randall-Sundrum model has been discussed in the framework of AdS/CFT correspondence $[14,15]$.

The motivation for the present study is to discuss the relevance of going beyond the infinite quartic coupling limit, in physical terms, and to explore its phenomenological implications. The plan of this paper is as follows: in Section 2, we describe the model and calculate the effective potential, its extremization condition, and the value of the stabilized modulus. In Section 3, we study the modulus for the case where the quartic coupling is finite but very large. We obtain the mass of the radion in this limit as well. Finally in Section 4 we present our conclusions.

\section{Review of the GW Mechanism}

In this section, mainly based on [6], we present a brief review of the GW mechanism in the limit of finite quartic coupling. The action of the model is of the form:

$$
S=S_{\text {gravity }}+S_{\text {vis }}+S_{\text {hid }}+S_{\Phi}
$$

where

$$
\begin{aligned}
S_{\text {gravity }}= & \int d^{4} x \int_{-\pi}^{\pi} d \phi \sqrt{G}\left[2 M^{3} R-\Lambda\right] \\
S_{\text {vis }}= & \int d^{4} x \sqrt{-g_{s}}\left[L_{s}-V_{s}\right] \\
S_{\text {hid }}= & \int d^{4} x \sqrt{-g_{p}}\left[L_{p}-V_{p}\right] \\
S_{\Phi}= & \frac{1}{2} \int d x^{4} \int_{-\pi}^{\pi} d \phi \sqrt{G}\left(G^{M N} \partial_{M} \Phi \partial_{N} \Phi-m^{2} \Phi^{2}\right) \\
& -\int d x^{4} \sqrt{-g_{s}} \lambda_{s}\left(\Phi^{2}-v_{s}^{2}\right)^{2} \\
& -\int d x^{4} \sqrt{-g_{p}} \lambda_{p}\left(\Phi^{2}-v_{p}^{2}\right)^{2}
\end{aligned}
$$

where $\Lambda$ is the five-dimensional cosmological constant, $V_{s}, V_{p}$ are the visible and hidden brane tensions, and $G=\operatorname{det}\left[G_{M N}\right]$.

The $\phi$-dependent vacuum expectation value $\Phi(\phi)$ is obtained from the equation of motion:

$$
\begin{aligned}
\partial_{\phi}\left(e^{-4 \sigma} \partial_{\phi} \Phi\right)= & m^{2} r^{2} e^{-4 \sigma} \Phi \\
& +4 e^{-4 \sigma} \lambda_{s} r \Phi\left(\Phi^{2}-v_{s}^{2}\right) \delta(\phi-\pi) \\
& +4 e^{-4 \sigma} \lambda_{p} r \Phi\left(\Phi^{2}-v_{p}^{2}\right) \delta(\phi),
\end{aligned}
$$

where $\sigma=k r_{c}|\phi|$. Away from the boundaries $(\phi=0, \pi)$ the solution is

$$
\Phi(\phi)=A e^{(\nu+2) \sigma}+B e^{(-v+2) \sigma}
$$

where $v=\sqrt{4+m^{2} / k^{2}} \approx 2+\epsilon$.
If we insert this solution in (5) and integrate over $\phi$ we obtain the effective 4-dimensional potential for the modulus $r$, namely, $V_{\Phi}(r)$ which is given by

$$
\begin{aligned}
V_{\Phi}(r)= & k(\nu+2) A^{2}\left(e^{2 v k r \pi}-1\right) \\
& +k(\nu-2) B^{2}\left(1-e^{-2 v k r \pi}\right) \\
& +\lambda_{s} e^{-4 k r \pi}\left(\Phi^{2}(\pi)-v_{s}^{2}\right)^{2} \\
& +\lambda_{p}\left(\Phi^{2}(0)-v_{p}^{2}\right)^{2} .
\end{aligned}
$$

The coefficients $A$ and $B$ are determined by imposing appropriate boundary conditions on the 3 branes. Putting (7) into (6) and matching delta functions, the conditions are obtained as

$$
\begin{aligned}
& k[(2+v) A+(2-v) B]-2 \lambda_{p} \Phi(0)\left[\Phi(0)^{2}-v_{p}^{2}\right]=0, \\
& k e^{2 k r \pi}\left[(2+v) e^{v k r \pi} A+(2-v) e^{-v k r \pi} B\right] \\
& \quad+2 \lambda_{s} \Phi(\pi)\left[\Phi(\pi)^{2}-v_{s}^{2}\right]=0 .
\end{aligned}
$$

For arbitrary value of $\lambda$ the boundary values of the scalar field at the two orbifold fixed points are $\Phi(\phi=0)=Q_{p}(r)$ and $\Phi(\phi=\pi)=Q_{s}(r)$. Now $A$ and $B$ can be written, from (7), in terms of boundary values of the scalar field as follows:

$$
\begin{aligned}
& A=\frac{Q_{s}(r) e^{-2 \sigma}-Q_{p}(r) e^{-v \sigma}}{2 \sinh (\nu \sigma)}, \\
& B=\frac{Q_{p}(r) e^{\nu \sigma}-Q_{s}(r) e^{-2 \sigma}}{2 \sinh (\nu \sigma)} .
\end{aligned}
$$

Putting above expressions for $A$ and $B$ into (9), we get

$$
\begin{aligned}
& \frac{v}{2 \sinh (\nu \sigma)}\left[e^{-2 \sigma}-\left(\frac{\nu+2}{2 \nu} e^{-v \sigma}+\frac{\nu-2}{2 \nu} e^{\nu \sigma}\right) \frac{Q_{p}}{Q_{s}}\right] \\
& =\frac{2 \lambda_{p}}{k} \frac{Q_{p}}{Q_{s}}\left(Q_{p}^{2}-v_{p}^{2}\right), \\
& \frac{v}{2 \sinh (\nu \sigma)}\left[\frac{Q_{p}}{Q_{s}}-\left(\frac{\nu+2}{2 v} e^{(\nu-2) \sigma}+\frac{v-2}{2 v} e^{-(\nu+2) \sigma}\right)\right] \\
& =\frac{2 \lambda_{s}}{k}\left(Q_{s}^{2}-v_{s}^{2}\right) e^{-2 \sigma} .
\end{aligned}
$$

Using (10) and (12) into the extremization condition for the effective potential $\left(d V_{\Phi}(r) / d r=0\right)$, the modulus can be obtained

$$
\begin{aligned}
k r_{ \pm} & =\frac{1}{\pi(\nu-2)} \\
& \cdot \ln \left[\frac{1}{\left((2+\nu) / 2 \nu+((\nu-2) / 2 \nu) e^{-2 v \sigma}\right)}\left(\frac{Q_{p}(r)}{Q_{s}(r)}\right)\right. \\
& \left.\cdot\left(\frac{1}{1 \pm C \sqrt{\lambda_{s} Q_{s}^{2}(r) /\left(1+\lambda_{s} Q_{s}^{2}(r)\right)}}\right)\right]
\end{aligned}
$$


where

$$
=\sqrt{1-\frac{4\left[(\nu+2) e^{2(\nu-2) \sigma}-e^{-4 \sigma}\left(4-v^{2}\right)+(2-\nu) e^{-2(\nu+2) \sigma}\right]}{\left[(2+\nu) e^{(\nu-2) \sigma}+(\nu-2) e^{-(\nu+2) \sigma}\right]^{2}} .}
$$

For $k r_{+}, d^{2} V / d r^{2}>0$, and for $k r_{-}, d^{2} V / d r^{2}<0$. So, $k r_{+}$ and $k r_{-}$are minimum and maximum of the potential, respectively. Clearly, $k r_{+}$and $k r_{-}$are, respectively, corresponding to stability and instability of the modulus field or the radion field.

\section{The Modulus and the Mass of Radion}

In this section we consider some observable quantities such as the modulus and the mass of radion.

3.1. The Modulus. Now we study the stable and unstable values of the modulus for the original GW mechanism at finite coupling. The analytic expression for the modulus was obtained and analyzed in [6], but we want to investigate the modulus by taking the parameter $\epsilon$ as a variable. In the large $k r$ limit, the values of the stable and unstable modulus when $\lambda_{p} \rightarrow \infty, \lambda_{s} \rightarrow \infty$ are

$$
k r_{ \pm}=\frac{1}{\pi(\nu-2)} \ln \left[\frac{2 \nu v_{p}}{\left(\nu+2 \pm \sqrt{v^{2}-4}\right) v_{s}}\right]
$$

In order to consider the value of the stabilized modulus for the large but finite value of the quartic coupling constant, $1 / \lambda$ expansion of boundary scalar field should be considered

$$
\begin{gathered}
Q_{p}(r)=v_{p}+\frac{k}{\lambda_{p} v_{p}} \frac{v e^{-2 \sigma}}{4 \sinh (\nu \sigma)}\left[\frac{v_{s}}{v_{p}}\right. \\
\left.-\left(\frac{2+v}{2 v} e^{(2-v) \sigma}+\frac{v-2}{2 \nu} e^{(\nu+2) \sigma}\right)\right], \\
Q_{s}(r)=v_{s}+\frac{k}{\lambda_{s} v_{s}} \frac{v e^{2 \sigma}}{4 \sinh (\nu \sigma)}\left[\frac{v_{p}}{v_{s}}\right. \\
\left.-\left(\frac{2+v}{2 v} e^{(\nu-2) \sigma}+\frac{v-2}{2 \nu} e^{-(\nu+2) \sigma}\right)\right] .
\end{gathered}
$$

The values of the modulus at finite value of the quartic coupling are [6]

$$
\begin{aligned}
k r_{ \pm} & =\frac{1}{\pi(v-2)} \ln \left[\frac{2 v}{2+v}\right. \\
& \cdot \frac{n}{1 \pm \sqrt{(v-2) /(v+2)}(1-q / 2)}\left(1-\frac{t(v-2)}{4}\right. \\
& \left.\left.+\frac{q(v+2)}{4}-\frac{q v n}{2} e^{(2-v) k \pi r_{ \pm}}\right)\right]
\end{aligned}
$$

where

$$
\begin{aligned}
& n=\frac{v_{p}}{v_{s}}, \\
& t=\frac{k}{\lambda_{p} v_{p}^{2}}, \\
& q=\frac{k}{\lambda_{s} v_{s}^{2}} .
\end{aligned}
$$

The values of the stable and unstable modulus $k r_{ \pm}$are obtained by solving (17) which are

$$
k r_{ \pm}=\frac{1}{\pi(\nu-2)} \ln \left[\frac{\alpha+\sqrt{\alpha^{2}-4 \beta}}{2}\right]
$$

where

$$
\begin{aligned}
& \alpha=\gamma_{ \pm}\left(1-\frac{t(\nu-2)}{4}+\frac{q(\nu+2)}{4}\right), \\
& \beta=\frac{\gamma_{ \pm} \nu q n}{2}, \\
& \quad \text { with } \gamma_{ \pm}=\frac{2 v n}{v+2} \frac{1}{1 \pm \sqrt{(v-2) /(v+2)}(1-q / 2)} .
\end{aligned}
$$

Using above equation, we can analyze the modulus $k r_{ \pm}$by taking the parameter $\epsilon$ as a variable. Figure 1 shows the variation of the stable modulus $k r_{+}$versus the parameter $\epsilon$. In this figure the value of $v_{s}=1$ and $v_{p}=1.2$. The solid curve corresponds to case of infinite coupling. The dashed curve corresponds to the case where $t=q=0.1$ and the dotted curve corresponds to the case where $t=q=0.2$. As seen above, $k r_{-}$is another value of the modulus which, because of $d^{2} V / d r^{2}<0$, corresponds to the maximum of the potential. Due to the maximality, $k r_{-}$leads to instability, that is, for any small perturbation the system will roll down to the minimum. Figure 2 shows the variation of the unstable modulus $k r_{-}$versus the parameter $\epsilon$. The value of $v_{s}=1$ and $v_{p}=1.2$. The solid curve corresponds to case of infinite coupling. The dashed curve corresponds to the case where $t=q=0.1$ and the dotted curve corresponds to the case where $t=q=0.2$. From Figures 1 and 2, we observe that by increasing the value of the parameters $t$ and $q$, we obtain more deviation from usual Randall-Sundrum case. As we know the value of $k r_{+} \sim 12$ may solve hierarchy problem. From this point of view, Figure 1 shows that the parameter $\epsilon$ in the finite coupling case can be smaller in comparison with the infinite coupling case.

It is appropriate to study the difference of the modulus at finite and infinite values of the quartic coupling constant; the result is

$$
\begin{aligned}
4 \pi & {\left[\left(k r_{+}\right)_{\infty}-\left(k r_{+}\right)_{f}\right] } \\
& =\frac{q}{\sqrt{\epsilon}}+\frac{1}{2}(q+2 t)+\frac{\sqrt{\epsilon} q}{8}+\cdots,
\end{aligned}
$$

where in the series expansion we have kept the linear terms of the parameters $t$ and $q$. 


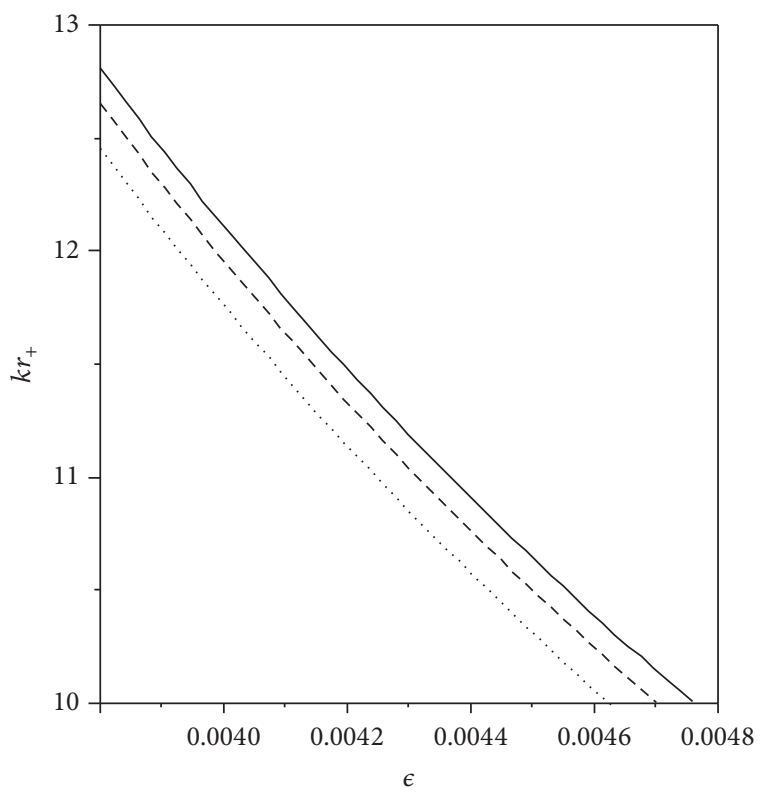

FIgURE 1: The variation of $k r_{+}$defined by (15) and (19) versus the parameter $\epsilon$. The values of the parameters are $v_{s}=1$ and $v_{p}=1.2$. The solid curve corresponds to limit of infinite coupling $(t=q=$ 0.0 ). For the dashed curve the values of these parameters are $t=q=$ 0.1 and for the dotted curve are $t=q=0.2$.

3.2. Mass of the Radion. The phenomenology of radion has been addressed in $[8,11,16]$. The canonically normalized radion field is $\Phi=f \widehat{\Phi}$, where $f=\sqrt{6 M^{3} / k}$ is another scale of order of Planck mass and

$$
\widehat{\Phi}=e^{-k \pi r} .
$$

The radion mass is defined the second derivative of $V_{\Phi}$ with respect to the canonically normalized radion field evaluated at its minimum. Hence

$$
\begin{aligned}
m_{\Phi}^{2} & =\left.\frac{d^{2} V_{\Phi}}{d \Phi^{2}}\right|_{\Phi=\Phi_{+}}=\left.\left[\left(\frac{d r}{d \Phi}\right)^{2}\left(\frac{d V_{\Phi}^{2}(r)}{d r^{2}}\right)\right]\right|_{r=r_{+}} \\
& =\left.\frac{1}{(f k \pi)^{2}}\left[e^{2 k r \pi}\left(\frac{d V_{\Phi}^{2}(r)}{d r^{2}}\right)\right]\right|_{r=r_{+}} .
\end{aligned}
$$

In order to calculate the mass of radion, we calculate the second derivative of the effective potential and we get

$$
\begin{aligned}
& \frac{d V_{\Phi}^{2}(r)}{d r^{2}}=-\frac{4 k \pi v e^{-2 \sigma}}{\sinh (v \sigma)}\left[\lambda_{p}\left(Q_{p}^{2}-v_{p}^{2}\right) Q_{p} Q_{s}^{\prime}\right. \\
& \quad+\lambda_{s}\left(Q_{s}^{2}-v_{s}^{2}\right) Q_{s} Q_{p}^{\prime} \\
& \left.\quad+2 \pi \lambda_{p} \lambda_{s}\left(Q_{s}^{2}-v_{s}^{2}\right)\left(Q_{p}^{2}-v_{p}^{2}\right) Q_{p} Q_{s}\right] .
\end{aligned}
$$

In the limit of $\lambda_{p} \rightarrow \infty, \lambda_{s} \rightarrow \infty, Q_{s}=v_{s}$, and $Q_{p}=v_{p}$, we find

$$
\left(m_{\Phi}^{2}\right)_{\infty}=\frac{8 k^{2} v_{p} v_{s} \sqrt{\epsilon^{3}} e^{-v \pi\left(k r_{+}\right)_{\infty}}}{3 M^{3}} .
$$

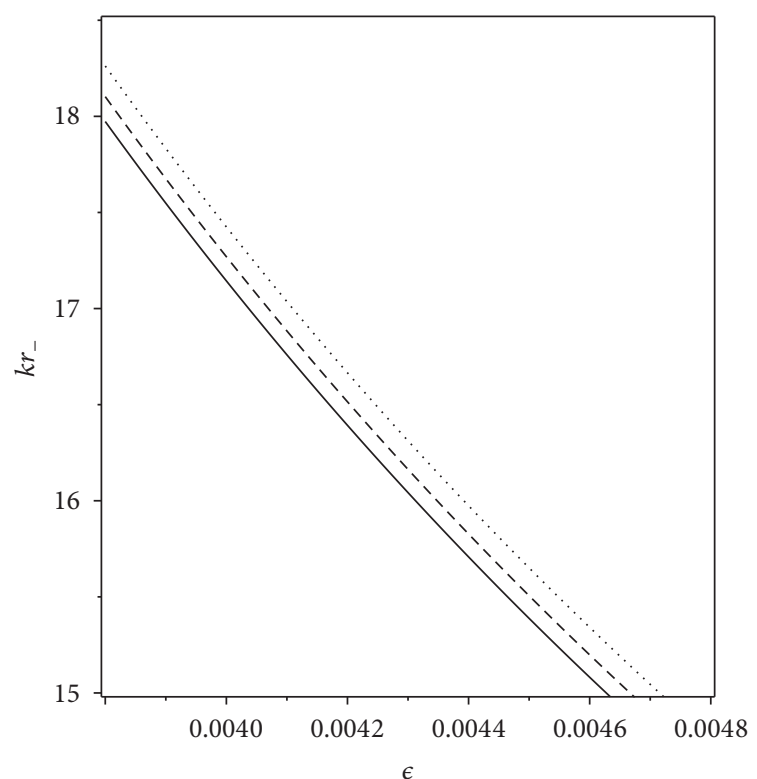

Figure 2: The variation of $k r_{-}$defined by (15) and (19) versus the parameter $\epsilon$. The values of the parameters are $v_{s}=1$ and $v_{p}=1.2$. The solid curve corresponds to limit of infinite coupling $(t=q=$ 0.0 ). For the dashed curve the values of these parameters are $t=q=$ 0.1 and for the dotted curve are $t=q=0.2$.

In [16] the scale factor is $f=\sqrt{24 M^{3} / k}$; moreover in their work $e^{\pi(\nu-2) k r}=n$. Now by using these values for the scale factor $f$ and the modulus $k r$, from (19) and (20), we obtain

$$
\left(m_{\Phi}^{2}\right)_{\infty}=\frac{k^{2} v_{s}^{2} \epsilon^{2} e^{-2 k \pi r}}{3 M^{3}},
$$

which is identical to the result of [16]. This in turn validates our results for the second derivative of the effective potential against a similar calculations presented in [6].

Similarly we can calculate the mass of radion in the case where the quartic coupling constants are finite; the result is

$$
\left(m_{\Phi}^{2}\right)_{f}=\frac{8 k^{2} Q_{p}(r) Q_{s}(r) \sqrt{\epsilon^{3}} e^{-v \pi\left(k r_{+}\right)_{f}}}{3 M^{3}} .
$$

Since $k \sim M_{p l} \sim M$ and $Q_{p}(r) \sim M_{p l}^{3 / 2} \sim Q_{s}(r)$, the radion mass $m_{\Phi}$ at finite coupling is $\mathcal{O}(\mathrm{TeV})$ when $k r_{+} \sim 12$. This is in agreement with previous works in the context of radion phenomenology. As seen above, the radion mass has dependency on the parameter $\epsilon \equiv m^{2} / 4 k^{2}$ as $\epsilon^{3 / 2}$. If one takes into account backreaction of the stabilizing field $\Phi$ on the background geometry, the radion mass turns out to have $\epsilon^{2}$ dependence [17]. This discrepancy of $\epsilon$ dependence between these two approaches may come from the fact that during all calculations we have assumed that $v \approx 2+\epsilon(\epsilon \ll 1)$ and we have neglected higher order terms of $\epsilon$. It would be of interest to take $v \approx 2+\epsilon-1 / 4 \epsilon^{2}$ and study similarities and differences between the effective potential and the exact gravity-scalar approaches. 


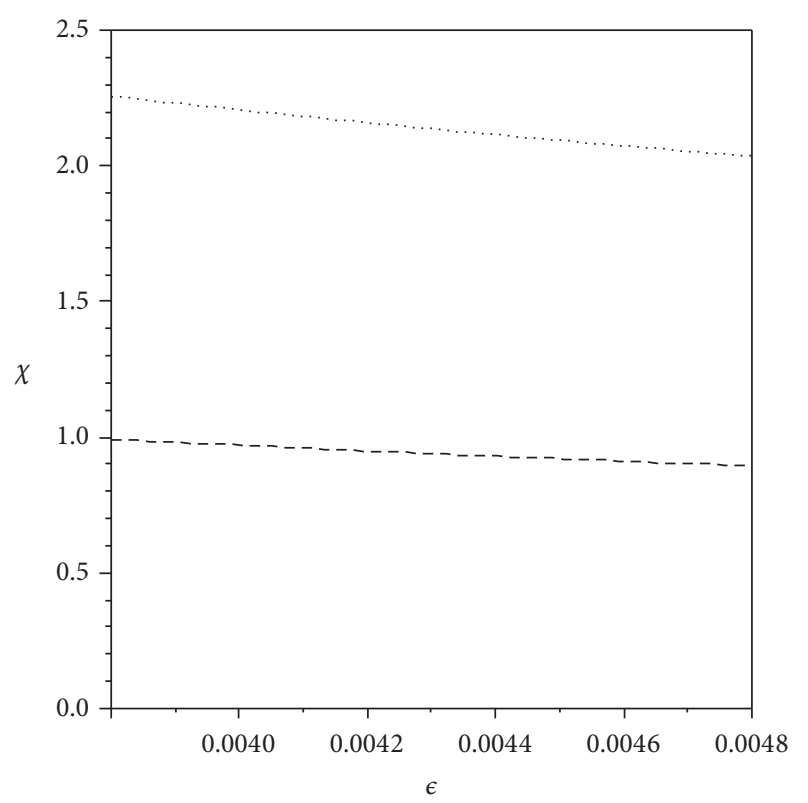

FIgURE 3: The variation of $\chi$ defined by (28) versus the parameter $\epsilon$. The values of the parameters are $v_{s}=1$ and $v_{p}=1.2$. For the dashed curve the values of the parameters are $t=q=0.1$ and for the dotted curve are $t=q=0.2$.

It is appropriate to study the logarithmic ratio of these masses which we denote by $\chi$ defined by

$$
\begin{aligned}
\chi & =\ln \left[\frac{\left(m_{\Phi}^{2}\right)_{f}}{\left(m_{\Phi}^{2}\right)_{\infty}}\right]=v \pi\left[\left(k r_{+}\right)_{\infty}-\left(k r_{+}\right)_{f}\right] \\
& =\frac{1}{2}\left[\frac{q}{\sqrt{\epsilon}}+\frac{1}{2}(q+2 t)+\frac{5 \sqrt{\epsilon} q}{8}+\cdots\right] .
\end{aligned}
$$

Figure 3 shows the variation of the $\chi$ versus the parameter $\epsilon$. In this figure the value of $v_{s}=1$ and $v_{p}=1.2$. The dashed curve corresponds to the case where $t=q=0.1$ and the dotted curve corresponds to the case where $t=q=0.2$. As seen in Figure 3 , the mass of the radion $\left(m_{\Phi}\right)_{f}$ at finite value of $\lambda$ could be much larger than the original value reported in [16] (the infinite coupling case). From phenomenological point of view, this is a reasonable result because it shows that the mass of the radion is governed by the strength of radion coupling. Moreover, since the radion mass increases as the finite quartic coupling becomes smaller, it could be considered in the context of high-mass radion or Higgsradion mixed scenarios [18-24].

\section{Conclusions}

We have made a critical assessment of the GW mechanism for the stabilization of modulus. We have managed to extend the work initiated in [6] to the phenomenology of radion. The limit studied by Goldberger and Wise, because the boundary conditions become very simple in this case, corresponds to the limit of infinite quartic coupling of the scalar potential term on the boundary branes. We have succeeded in refining this aspect of the mechanism. We have found correction for the value of the modulus and the mass of the radion. Instead of brane potential with quartic coupling, it is also possible to consider brane potential of the quadratic form. We plan to report on these issues in the future. As final note, it is possible to stabilize the modulus by a massless scalar nonminimally coupled to gravity. In such work, it would be of interest to study the limit of finite coupling.

\section{Conflicts of Interest}

The authors declare that there are no conflicts of interest regarding the publication of this paper.

\section{References}

[1] L. Randall and R. Sundrum, "Large mass hierarchy from a small extra dimension," Physical Review Letters, vol. 83, no. 17, pp. 3370-3373, 1999.

[2] W. D. Goldberger and M. B. Wise, "Modulus Stabilization with Bulk Fields," Physical Review Letters, vol. 83, no. 24, article 4922, 1999.

[3] B. Grzadkowski and J. F. Gunion, "Bulk scalar stabilization of the radion without metric back reaction in the RandallSundrum model," Physical Review D, vol. 68, no. 5, Article ID 055002, 2003.

[4] L. N. Granda and A. Oliveros, "Modulus stabilization via nonminimal coupling," Europhysics Letters, vol. 74, no. 2, pp. 236239, 2006.

[5] A. Tofighi and M. Moazzen, "Stabilization of modulus in randall-sundrum model I by bulk scalar fields," Modern Physics Letters A. Particles and Fields, Gravitation, Cosmology, Nuclear Physics, vol. 28, no. 11, Article ID 1350044, 2013.

[6] A. Dey, D. Maity, and S. SenGupta, "Critical analysis of Goldberger-Wise stabilization of the Randall-Sundrum braneworld scenario," Physical Review D, vol. 75, no. 10, Article ID 107901, 2007.

[7] T. Tanaka and X. Montes, "Gravity in the brane-world for twobranes model with stabilized modulus," Nuclear Physics B, vol. 582, no. 1-3, pp. 259-276, 2000.

[8] C. Csaki, M. Graesser, L. Randall, and J. Terning, "Cosmology of brane models with radion stabilization," Physical Review. D. Third Series, vol. 62, no. 4, Article ID 045015, 2000.

[9] K. Ghoroku and A. Nakamura, "Stability of Randall-Sundrum brane-world and tachyonic scalar," Physical Review D, vol. 64, no. 8, Article ID 084028, 2001.

[10] J. Lesgourgues and L. Sorbo, "Goldberger-Wise variations: stabilizing brane models with a bulk scalar," Physical Review D, vol. 69, no. 8, Article ID 084010, 2004.

[11] J. M. Cline and H. Firouzjahi, "Brane-world cosmology of modulus stabilization with a bulk scalar field," Physical Review $D$, vol. 64, no. 2, Article ID 023505, 2001.

[12] P. Kanti, K. A. Olive, and M. Pospelov, "On the stabilization of the size of extra dimensions," Physics Letters B, vol. 538, no. 1-2, pp. 146-158, 2002.

[13] N. Haba, K. Oda, and R. Takahashi, "Dirichlet Higgs as radion stabilizer in warped compactification," Journal of High Energy Physics, vol. 2011, no. 5, article 125, 2011.

[14] Z. Chacko, R. K. Mishra, and D. Stolarski, "Dynamics of a stabilized radion and duality," Journal of High Energy Physics, vol. 2013, article 121, 2013. 
[15] Z. Chacko, R. K. Mishra, D. Stolarski, and C. B. Verhaaren, "Interactions of a stabilized radion and duality," Physical Review D-Particles, Fields, Gravitation and Cosmology, vol. 92, no. 5, Article ID 056004, 2015.

[16] W. D. Goldberger and M. B. Wise, "Phenomenology of a stabilized modulus," Physics Letters B, vol. 475, no. 3-4, pp. 275279, 2000.

[17] C. Csáki, M. L. Graesser, and G. D. Kribs, "Radion dynamics and electroweak physics," Physical Review D, vol. 63, no. 6, Article ID 065002, 2001.

[18] A. Ahmed, B. M. Dillon, B. Grzadkowski, J. F. Gunion, and Y. Jiang, "Higgs-radion interpretation of $750 \mathrm{GeV}$ di-photon excess at the LHC," https://arxiv.org/abs/1512.05771.

[19] P. Cox, A. D. Medina, T. S. Ray, and A. Spray, "Implications of diphoton searches for a radion in the bulk-Higgs scenario," International Journal of Modern Physics A, vol. 32, no. 4, Article ID 1750020, 2017.

[20] D. Bardhan, D. Bhatia, A. Chakraborty, U. Maitra, S. Raychaudhuri, and T. Samui, "Radion candidate for the LHC diphoton resonance," 2015, https://arxiv.org/abs/1512.06674.

[21] H. Davoudiasl and C. Zhang, "750 GeV messenger of dark conformal symmetry breaking," Physical Review D-Covering Particles, Fields, Gravitation, and Cosmology, vol. 93, no. 5, Article ID 055006, 2016.

[22] E. E. Boos, V. E. Bunichev, and I. P. Volobuev, "Can the $750 \mathrm{GeV}$ diphoton LHC excess be due to a radion-dominated state?" https://arxiv.org/abs/1603.04495.

[23] F. Abu-Ajamieh, R. Houtz, and R. Zheng, "Interpretation of $750 \mathrm{GeV}$ diphoton resonance within the RS model and the associated radion phenomenology," https://arxiv.org/abs/1607 .01464 .

[24] A. Chakraborty, U. Maitra, S. Raychaudhuri, and T. Samui, "Mixed Higgs-Radion states at the LHC-a detailed study," https://arxiv.org/abs/1701.07471. 

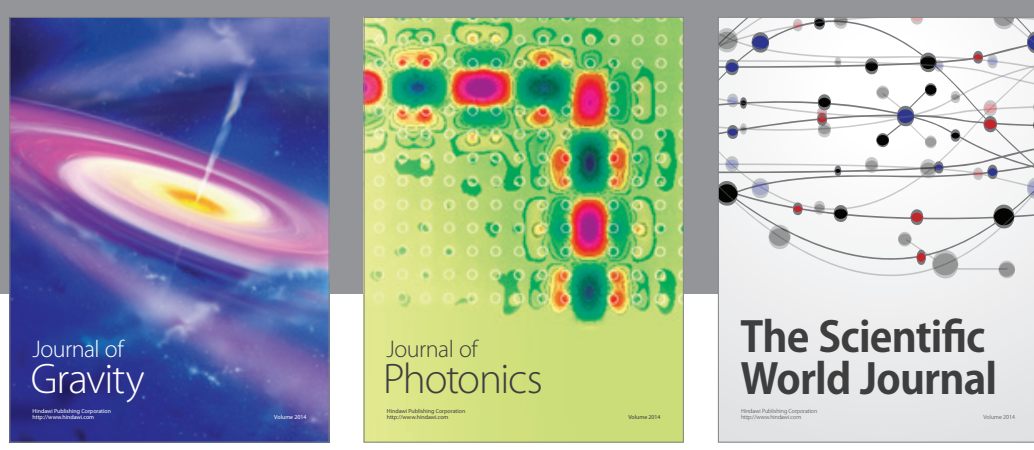

The Scientific World Journal
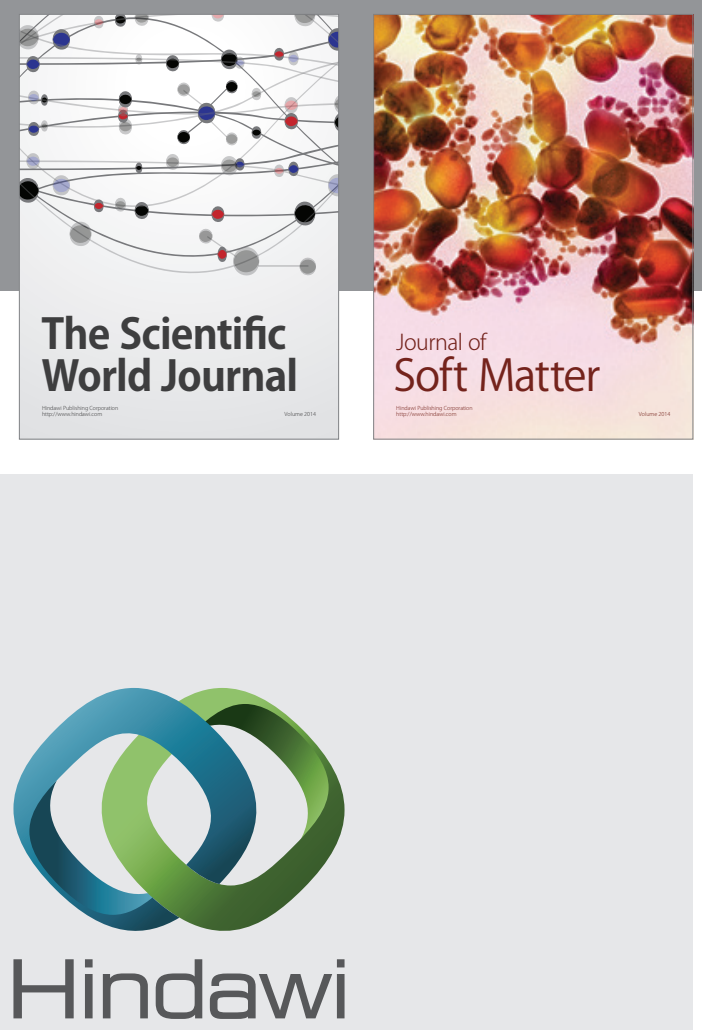

Submit your manuscripts at

https://www.hindawi.com
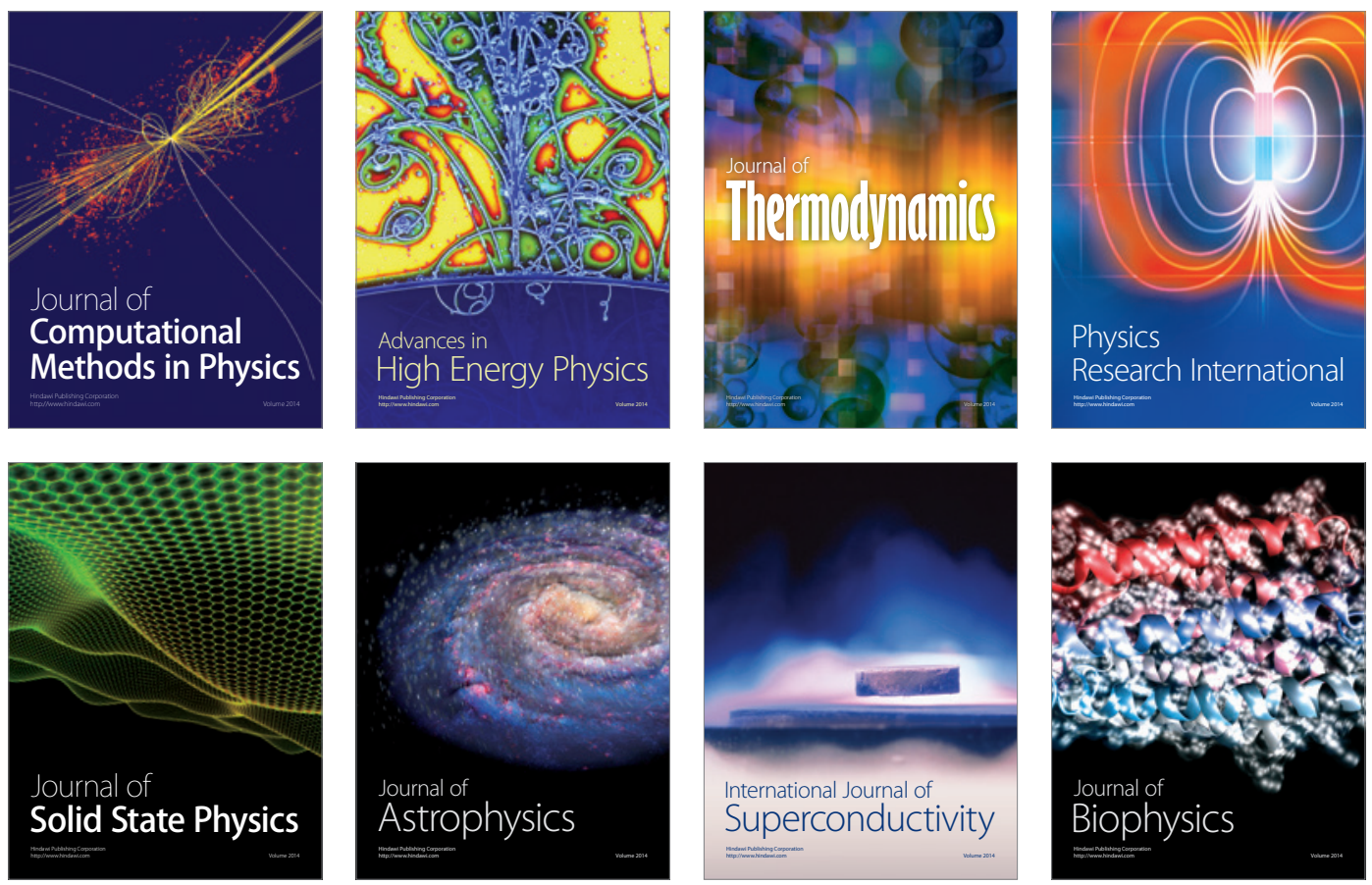
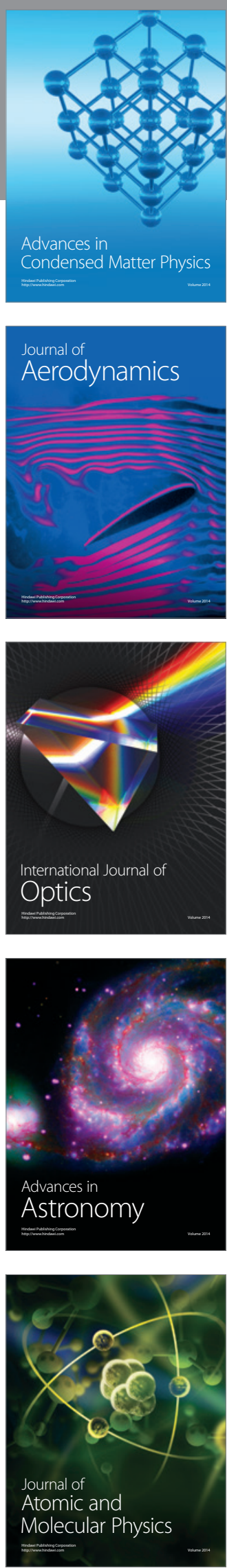